\title{
EHMTI-0067. Chronic headaches in female patients with irritable bowel syndrome: just another functional condition?
}

\author{
D Georgescu ${ }^{1 *}$, C Georgescu², LA Georgescu ${ }^{3}$ \\ From 4th European Headache and Migraine Trust International Congress: EHMTIC 2014 \\ Copenhagen, Denmark. 18-21 September 2014
}

\section{Introduction}

Among comorbidities associated to irritable bowel syndrome (IBS) there are headaches including migraine attacks, frequently reported in female gender.

\section{Aim}

Assessment of vascular involvement of migraine without aura in female patients with IBS.

\section{Methods}

30 female patients, mean age $=47,47 \pm 8,38$ years, previously diagnosed with IBS (Rome II criteria), 15 predominant constipation(IBS-C) and 15 predominant diarrhea(IBS-D), complaining of mild to moderate migraine without aura (according to International Headache Society criteria), with no records of cigarettes smoking, hypertension, diabetes mellitus, obesity or treatment requiring dyslipidemia, undertook Duplex carotidian examination with a linear 12 $\mathrm{MHz}$ transductor, GE Loqic 5 Expert high resolution ultrasound device. We assessed intima-media thickness (IMT) at $1 \mathrm{~cm}$ before right and left common carotid artery (CCA) bifurcation at the posterior wall and presence and severity of atherosclerotic plaques.

\section{Results}

12 patients(40\%) exhibited no vasular issues, IMT normal (6: IBS-D and 6: IBS-C); 18 patients (60\%) showed various aspects of endotelial dysfunction: abnormal IMT in 15 patients (8: IBS-D,7: IBS-C) and small nonstenotic, stable plaques in 3 patients (1:IBS-D, 2 :IBS-C) with fibrolipidic features. There was no statistically difference between the two groups neither having vs not having vascular issues $(\mathrm{p}=0,8974)$, nor between abnormal IMT vs presence of atherosclerotic plaques $(\mathrm{p}=0,5549)$.

\section{Conclusions}

More than half of IBS female patients complaining of migraine without aura presented features of endotelial dysfunction: either increasesd IMT or patent signs of early atherosclerotic plaques. No statistically significant differences concerning vascular issues were set between the two groups: IBS-C vs IBS-D patients.

No conflict of interest.

\section{Authors' details}

"Internal Medicine, "Victor Babes" University of Medicine and Pharmacy, Timisoara, Romania. ${ }^{2}$ Neurology, Academic Emergency Hospital, Timisoara, Romania. ${ }^{3}$ General Surgery, Academic Emergency Hospital, Timisoara, Romania.

Published: 18 September 2014

doi:10.1186/1129-2377-15-S1-D20

Cite this article as: Georgescu et al:: EHMTI-0067. Chronic headaches in female patients with irritable bowel syndrome: just another functional condition? The Journal of Headache and Pain 2014 15(Suppl 1):D20. 